\title{
Agomelatine versus Sertraline: An Observational, Open-labeled and 12 Weeks Follow-up Study on Efficacy and Tolerability
}

\author{
Esma Akpınar ${ }^{1}$, Cem Cerit ${ }^{2}$, Anıl Talas ${ }^{3}$, Ümit Tural ${ }^{2}$ \\ ${ }^{1}$ Psychiatry Clinic, Tokat Mental Health Hospital, Tokat, ${ }^{2}$ Department of Psychiatry, School of Medicine, Kocaeli University, Kocaeli, \\ ${ }^{3}$ Psychiatry Clinic, Tuzla State Hospital, Istanbul, Turkey
}

\begin{abstract}
Objective: In this open-labeled, 12 weeks follow-up study, we aimed to compare the efficacy and tolerability of agomelatine with sertraline

Methods: The outpatients of adult psychiatry clinic who have a new onset of depression and diagnosed as 'major depressive episode' by clinician according to the Diagnostic and Statistical Manual of Mental Disorders 4th edition and prescribed agomelatine (25 mg/day) or sertraline (50 mg/day) were included in the study.

Results: The decline of mean Montgomery-Asberg Depression Rating Scale (MADRS) scores of agomelatine group was significantly higher than the sertraline group at the end of 2nd week; however, the difference was not significant at the end of 3 months. Mean Clinical Global Impression-Improvement scale (CGI-I) scores of agomelatine group was lower than sertraline group at first week. Mean CGI-Severity scale and CGI-I scores were favour to sertraline group at the end of the study. Remission rates were $46.7 \%$ for sertraline group and $33.3 \%$ for agomelatine group while response rates were $76.7 \%$ for both groups. Any patient from agomelatine group dropped-out due to adverse effects. The amount of side effects was also less with agomelatine. Conclusion: Agomelatine has a rapid onset efficacy on depressive symptoms and this can be beneficial for some critical cases. Considering MADRS scores, agomelatine seems to have similar efficacy with sertraline but we also point the need for long term studies since CGI scores were favour to sertraline group at the end of the study. Agomelatine has a favourable tolerability profile both in terms of discontinuation and the amount of side effects compared to sertraline.
\end{abstract}

KEY WORDS: Agomelatine; Sertraline; Clinical efficacy; Drug tolerance; Comparative study.

\section{INTRODUCTION}

Agomelatine is a melatonergic (MT1 and MT2 agonist) antidepressant with additional serotonin (5-HT-2C) antagonist properties. ${ }^{1)}$ This represents a novel pharmacology among antidepressants as agomelatine is the only antidepressant with melatonergic properties. The efficacy of agomelatine has been demonstrated at doses of 25-50 mg in patients with moderate to severe major depressive disorder (MDD). ${ }^{2,3)}$

There are a few studies comparing agomelatine with other antidepressants. In a comprehensive review of studies comparing agomelatine with selective serotonin reuptake inhibitor (SSRI) or venlafaxine, Guaiana et al. ${ }^{4)}$ re-

\footnotetext{
Received: March 2, 2016 / Revised: April 15, 2016

Accepted: April 25, 2016

Address for correspondence: Cem Cerit, MD

Department of Psychiatry, School of Medicine, Kocaeli University, Umuttepe Yerleșkesi 41380, İzmit/Kocaeli, Turkey

Tel: +90-2623037508, Fax: +90-2623037079

E-mail: cemcerit@yahoo.com
}

vealed that agomelatine did not provide a significant advantage in efficacy but was better tolerated than paroxetine and venlafaxine. However some of the studies in this review were unpublished and also most of them were sponsored by the pharmaceutical company. Based on a pooled analysis of four randomized clinical trials of agomelatine versus escitalopram, fluoxetine and sertraline, Demyttenaere et al. ${ }^{5)}$ concluded that agomelatine is at least as efficacious as SSRIs with a trend to fewer discontinuations due to adverse events. A randomised, double-blind study comparing efficacy and tolerability of agomelatine with fluoxetine showed that agomelatine and fluoxetine were equally effective in the treatment of MDD-associated symptoms and agomelatine showed good tolerability profile and safety. ${ }^{6)}$ In a long term (24 week), randomized, controlled and double-blind study comparing agomelatine with escitalopram, it is reported that agomelatine provides comparable antidepressant efficacy with better safety and tolerability profile and favourable effetcs on sleep-awake conditions. ${ }^{7}$

(c) This is an Open-Access article distributed under the terms of the Creative Commons Attribution Non-Commercial License (http://creativecommons.org/licenses/by-nc/4.0) which permits unrestricted non-commercial use, distribution, and reproduction in any medium, provided the original work is properly cited. 
In our opinion, studies on efficacy and tolerability of agomelatine is not sufficient so far and more comparative studies with widely used antidepressants is needed. In this open-labeled, 12 weeks follow-up study, we aimed to compare the efficacy and tolerability of agomelatine with a widely used antidepressant sertraline.

\section{METHODS}

\section{Study Design and Materials}

This study was carried out in the outpatient clinic of Haydarpasa Numune Training and Research Hospital between February 2013 and March 2014. Firstly, ethical comittee approval was obtained from the institute (HNEAH-KAEK-2012/85). Among the outpatients admitted to adult psychiatry clinic who have diagnosed as "major depressive episode" by clinician according to Diagnostic and Statistical Manual of Mental Disorders 4th edition and prescribed agomelatine $(25 \mathrm{mg}$ /day) or sertraline $(50 \mathrm{mg} /$ day) were included in the study. The other inclusion criteria were; to have a new onset depression and using none of psychotropic drugs recently (at least in the last month), to have none of chronic physical diseases, mental retardation and comorbid psychiatric diseases. Pregnant and puerperal patients and patients with suicidal ideas were also excluded from the study. The patients who accepted to participate in the study and signed written informed consent were included in the study. A second clinician, who is blind for the drug, performed the assessments of the study. Thereby first clinician maintained the clinical follow-up while second clinician maintained the assessments.

On the first interview, efficacy assessments were performed with Montgomery-Asberg Depression Rating Scale (MADRS), ${ }^{8)}$ Turkish version ${ }^{9)}$ and Clinical Global Impression (CGI) scale. ${ }^{10)}$ Further assessments were performed at 1st, 2nd, 4th, 8th and 12th weeks of treatment. The UKU (Udvalget for Kliniske Undersegelser) Side Effect Rating Scale was used to assess the tolerability of drugs. ${ }^{11)}$

During the follow-up period 6 patients (4 patients from sertraline group and 2 patients from agomelatine group) who did not come to subsequent interviews and did not respond to the phone call excluded from the study. The study was terminated when the total number of patients completed the study is reached to 30 in both groups.

\section{Statistics}

All statistical assessments were performed with IBM SPSS Statistics ver. 20.0 program (IBM Co., Armonk,
NY, USA). Rates and frequencies are reported as raw numbers and percentages. We used chi-square tests with continuity correction for $2 \times 2$ tables to examine for possible differences in the categorical variables. Fisher's exact test was used instead of chi-square test when a $2 \times 2$ table had a cell with an expected frequencyof less than 5 . We used $t$-tests for independent groups to evaluate differences in the continuous variables and also Mann-Whitney $U$-test is used regarding the distribution of continuous variables. After checking the preliminary assumptions, repeated measures analysis of variance (ANOVA) with simple contrasts was performed on six consecutive measurements of MADRS to explore the main (group) and interaction (time by group) differences. Following a significant global $\mathrm{F}$ test, univariate ANOVAs with Greenhouse-Geisser adjustment was then used to assess the statistical significance if sphericity could not be assumed. Statistical significance is accepted as $p<0.05$.

\section{RESULTS}

The initial doses were $25 \mathrm{mg}$ /day for agomelatine and $50 \mathrm{mg} /$ day for sertraline. The mean doses at the end of follow-up period was $30.83 \pm 10.75$ for agomelatine group and $59.16 \pm 17.95$ for sertraline group which were both close to the initial doses. Three patients of sertraline group dropped-out from the study due to adverse effects. Two of them were due to gastrointestinal adverse effects like nausea and dyspepsia and one was due to sleeplessness. Any patient from agomelatine group dropped-out from the study due to adverse effects.

Comparison of demographical and clinical data of patients using sertraline or agomelatine is presented in Table

Table 1. Comparison of demographical and clinical data of patients using sertraline or agomelatine

\begin{tabular}{lccc}
\hline \multicolumn{1}{c}{ Variable } & $\begin{array}{c}\text { Sertraline } \\
(\mathrm{n}=30)\end{array}$ & $\begin{array}{c}\text { Agomelatine } \\
(\mathrm{n}=30)\end{array}$ & $p$ value \\
\hline Age (yr) & $\begin{array}{c}35.16 \pm 12.08 \\
(21-63)\end{array}$ & $\begin{array}{c}34.16 \pm 8.84 \\
(20-61)\end{array}$ & 0.716 \\
& $21: 9$ & $23: 7$ & 0.559 \\
Sex (female:male) & & & 0.602 \\
Marital status & 16 & 18 & \\
$\quad$ Married & 14 & 12 & \\
$\quad$ Not married & 17 & 12 & 0.196 \\
Occupation & 13 & 18 & \\
$\quad$ Employeed & $7.73 \pm 4.66$ & $7.96 \pm 4.49$ & 0.844 \\
$\quad$ Not employeed & $0.66 \pm 0.71$ & $0.90 \pm 0.92$ & 0.278 \\
Education (yr) & & & \\
Past depression episodes & &
\end{tabular}

Values are presented as mean \pm standard deviation (range) or number only. 
1. There were no difference between the groups in terms of age, sex, marital status, education level, occupation and the number of past depression episodes.

Comparison of CGI-Severity scale (CGI-S) and CGIImprovement scale (CGI-I) scores of patient groups is shown in Table 2. Considering CGI-S scores, the only difference between sertraline and agomelatine groups was found at the third month, that the mean CGI-S scores of agomelatine group was higher than sertraline group. Mean CGI-I scores of sertraline group was higher than agomelatine group at the first week. However this finding reversed at the 8th week and mean CGI-I scores of agomelatine group was higher than sertraline group at the 8th and 12 th weeks.

MADRS scores $\leq 8$ is considered as 'remission', while more than $50 \%$ decline in MADRS score is considered as 'response' in this study. Comparison of remission and response rates of the study groups at the end of the study is shown in Table 3. Although the number of remitted patients in sertraline group was more than agomelatine group, the difference was not statistically significant. The number of patients responded to the treatment was equal across the groups.

Table 2. Comparison of mean CGI scores of the study groups

\begin{tabular}{cccc}
\hline & $\begin{array}{c}\text { Sertraline } \\
(n=30)\end{array}$ & $\begin{array}{c}\text { Agomelatine } \\
(n=30)\end{array}$ & $p$ value \\
\hline $\begin{array}{c}\text { CGI-S score } \\
\text { Week 0 }\end{array}$ & $4.00 \pm 0.58$ & $4.06 \pm 0.52$ & 0.647 \\
1 & $3.83 \pm 0.69$ & $3.73 \pm 0.78$ & 0.837 \\
2 & $3.76 \pm 0.81$ & $3.36 \pm 0.76$ & 0.106 \\
4 & $3.26 \pm 0.94$ & $3.16 \pm 0.91$ & 0.801 \\
8 & $2.73 \pm 1.01$ & $2.93 \pm 0.78$ & 0.449 \\
12 & $2.00 \pm 0.90$ & $2.66 \pm 0.95$ & 0.008 \\
CGI-I score & & & \\
Week 1 & $3.66 \pm 0.47$ & $3.33 \pm 0.60$ & 0.015 \\
2 & $3.26 \pm 0.52$ & $3.16 \pm 0.53$ & 0.473 \\
4 & $2.83 \pm 0.53$ & $3.06 \pm 0.78$ & 0.278 \\
8 & $2.46 \pm 0.62$ & $2.96 \pm 0.76$ & 0.004 \\
12 & $1.96 \pm 0.76$ & $2.76 \pm 1.07$ & 0.002 \\
\hline
\end{tabular}

Values are presented as mean \pm standard deviation.

CGI, Clinical Global Impression; CGI-S, CGI-Severity scale; CGI-I, CGI-Improvement scale.

Table 3. Comparison of remission and response rates of the study groups

\begin{tabular}{lccc}
\hline \multicolumn{1}{c}{ Patient } & $\begin{array}{c}\text { Sertraline } \\
(n=30)\end{array}$ & $\begin{array}{c}\text { Agomelatine } \\
(n=30)\end{array}$ & p value \\
\hline Remitted/nonremitted $(n)$ & $14 / 16$ & $10 / 20$ & 0.292 \\
Remission rate (\%) & 46.7 & 33.3 & \\
Responded/nonresponded & $23 / 7$ & $23 / 7$ & 1 \\
Response rate (\%) & 76.7 & 76.7 & \\
\hline
\end{tabular}

Change of mean MADRS scores of the study groups in 3 months period has shown in Figure 1. Mean MADRS scores of agomelatine and sertraline groups both decreased significantly at the end of 3 months follow-up ( $\mathrm{F}=260.97$, degree of freedom $[\mathrm{df}]=1, p<0.001$ for sertraline and $\mathrm{F}=455.96, \mathrm{df}=1, p<0.001$ for agomelatine). The time by group interaction in MADRS scores was not significant at the end of 3 months follow-up $(\mathrm{F}=0.005, \mathrm{df}=1$, $p=0.945)$. However the mean MADRS scores of agomelatine group significantly more declined from baseline than the sertraline group at the end of 2 nd week $(F=10.19$, $\mathrm{df}=1, p=0.002)$. This difference disappared at the end of 4 th week $(\mathrm{F}=1.954, \mathrm{df}=1, p=0.167)$ and it has proceeded

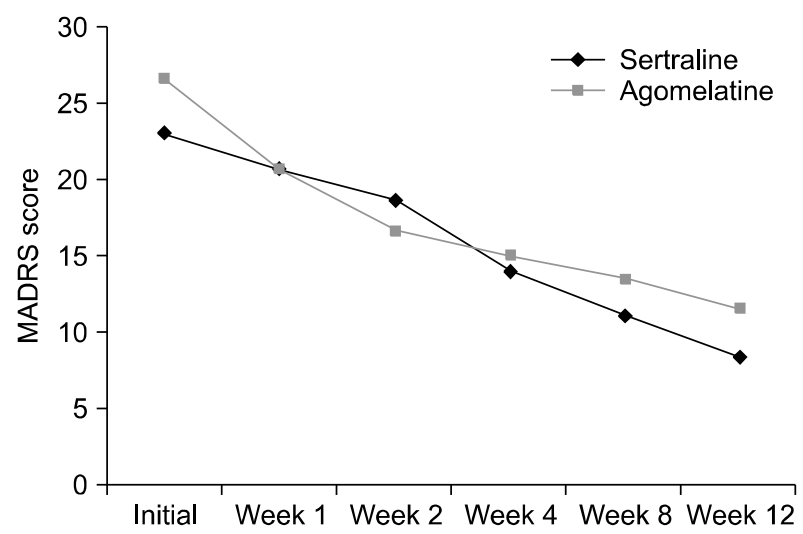

Fig. 1. Change of mean Montgomery-Asberg Depression Rating Scale (MADRS) scores of the study groups in 3 months period.

Table 4. Comparison of the side effects between study groups during the follow-up period

\begin{tabular}{cccc}
\hline $\begin{array}{c}\text { UKU Side } \\
\text { Effects Scale }\end{array}$ & $\begin{array}{c}\text { Sertraline } \\
\text { group }(n=30)\end{array}$ & $\begin{array}{c}\text { Agomelatine } \\
\text { group }(n=30)\end{array}$ & p value \\
\hline Week 1 & $2.06 \pm 1.83$ & $1.56 \pm 1.54$ & 0.259 \\
Psychiatric & $0.13 \pm 0.34$ & $0.06 \pm 0.25$ & 0.398 \\
Neurologic & $2.33 \pm 1.66$ & $1.10 \pm 1.21$ & 0.002 \\
Autonomic & $0.76 \pm 0.93$ & $0.50 \pm 0.73$ & 0.224 \\
Other & $8.23 \pm 3.64$ & $6.10 \pm 2.86$ & 0.015 \\
Total & $1.36 \pm 1.54$ & $0.96 \pm 1.40$ & 0.297 \\
Week 4 & $0.06 \pm 0.25$ & $0.03 \pm 0.18$ & 0.562 \\
Psychiatric & $1.73 \pm 1.38$ & $1.26 \pm 1.25$ & 0.178 \\
Neurologic & $0.90 \pm 0.84$ & $0.66 \pm 1.26$ & 0.406 \\
Autonomic & $7.03 \pm 2.79$ & $5.66 \pm 3.02$ & 0.074 \\
Other & & & \\
Total & $0.76 \pm 1.13$ & $0.20 \pm 0.40$ & 0.014 \\
Week 12 & - & - & - \\
Psychiatric & $1.33 \pm 1.47$ & $0.40 \pm 0.81$ & 0.004 \\
Neurologic & $0.63 \pm 0.76$ & $0.26 \pm 0.63$ & 0.049 \\
Autonomic & $5.33 \pm 2.98$ & $3.30 \pm 1.68$ & 0.004 \\
Other & & & \\
Total & & & \\
\hline
\end{tabular}

Values are presented as mean \pm standard deviation. UKU, Udvalget for Kliniske Undersegelser. 
to the end of the follow-up.

Mean MADRS scores of sertraline and agomelatine groups were $23.23 \pm 6.51$ and $26.63 \pm 5.24$ respectively at the beginning of the study $(p=0.030)$ and $8.40 \pm 5.46$ and $11.66 \pm 6.94$ respectively at the end of the study ( $p=0.048)$. The mean MADRS score of agomelatine group was higher than sertraline group both at the beginning and at the end of the study.

The comparison of side effects between study groups during the follow-up period has shown in Table 4. The sum of side effect scores at first week was higher in sertraline group than agomelatine group $(p=0.015)$. This was mainly due to the more apparent autonomic side effects in sertraline group like reduced salivation (dry mouth), palpitations and increased sweating. Although the sum of psychiatric side effects were similar between groups, some psychiatric side effects like astenia and reduced sleep duration was higher in sertraline group $(p=0.020$ and $p=0.024$, respectively) at first week. The sum of side effect scores tend to decrease at 4th week in both groups and there were no significant difference between groups at 4 th week ( $p=0.074)$. However, even side effect scores maintained decreasing in both groups, all group of side effects (except neurological side effects) were higher in sertraline group than agomelatine group at the end of 3 months period. There were no neurologic side effect in both groups at the end of the follow-up. Even it was rare, the main side effects in agomelatine group was sedation and increased duration of sleep particularly at the first week.

\section{DISCUSSION}

Most of the comperative studies of agomelatine put forth similar efficacy, ${ }^{7,12-14)}$ while some of the studies demonstrate superior efficacy ${ }^{15,16)}$ of agomelatine to other commonly used antidepressants. Considering the efficacy data in this study, we found that mean MADRS scores of patients using agomelatine rapidly decreased in the first 2 weeks. However patients using sertraline respond the drug mainly after 2 weeks. Moreover better improvement scores with agomelatine has found in CGI-I in the first week. This early difference may be due to the well-known favourable effect of agomelatine on sleep related parameters. ${ }^{17)}$ This rapid effect on efficacy may be crucial for some critical situations like patients with suicidal tendencies. Nevertheless considering MADRS scores, the efficacy score of sertraline group was similar to agomelatine group at the end of first month and there were no difference in CGI-S and CGI-I scores between groups at that point. There were no difference between groups at the end of the study in terms of decline in MADRS scores. However considering CGI scores, sertraline group tend to demonstrate better improvement particularly after 2 nd month. It is difficult to comment if the long term efficacy of sertraline would be better than agomelatine with this result because this finding is not confirmed with MADRS scores in this study and the duration of the study was not that long. Therefore in this point we may just highlight the need for long term studies to make a healthy interpretation. Although it was not statistically significant, the remission rate of sertraline group (46.7\%) was higher than agomelatine group (33.3\%) at the end of the study while the response rates were equal across groups (76.7\%).

Most studies comparing agomelatine to other drugs have used Hamilton Rating Scale for Depression (HAM-D) scale for efficacy. ${ }^{6,13,14,16,18,19)}$ In our opinion using MADRS or HAM-D is an other important issue that the percentage of items on sleep in HAM-D is more than in MADRS. It is very well documented that agomelatine improves sleep quality ${ }^{7,20,21)}$ and such differences may be considered when commenting on results. In a meta-analyses of randomized clinical trials of agomelatine comparing with other antidepressants, Huang et al. ${ }^{21)}$ revealed that even agomelatine shows a superior efficacy to other antidepressants, the difference in efficacy is not considered clinically relevant because of agomelatine's unique effect on sleep-awake conditions. We also support that idea and we believe that more data is needed to comment on particularly the long term efficacy of agomelatine.

Most of the clinical studies with agomelatine evidence the favourable tolerability profile of agomelatine in patients with depression. ${ }^{6,16,22-25)}$ In our opinion tolerability is the strong side of agomelatine. Kennedy et al. ${ }^{12)}$ revealed that the discontinuation due to adverse events was $2.2 \%$ with agomelatine in their study sample. It was remarkable that there was no patient dropped-out due to side effects in agomelatine group in our study. Some side effects mainly related with increased sedation were noticeable in agomelatine group particularly in the first week. At first week, psychiatric and autonomic side effects like astenia and reduced sleep duration, dry mouth, palpitations and increased sweating was higher in sertraline group. However two of three drop-outs from sertraline group were due to gastrointestinal side effects like nausea and dyspepsia. All side effects maintained decreasing in both groups during the follow-up period but it was remakable that almost all group of side effects (psychiatric, autonomic and other) were less in agomelatine group than sertra- 
line group at the end of the follow-up and there was no neurological side effect in both groups. With this findings we assume that agomelatine has a spectacular tolerability profile and this profile seems to emerge even better during the later course of treatment.

In conlusion, comparing to sertraline, agomelatine has a rapid onset efficacy on depressive symptoms propably related with the improvement of sleep in patients with MDD and this can be beneficial for some particular cases. According to MADRS scores of this 3 months follow-up study agomelatine seems to have similar efficacy with sertraline. However considering CGI scores, we may suggest that studies investigating long term effects of agomelatine particularly on the core symptoms of depression other than vegetative symptoms would be beneficial. Agomelatine has a favourable tolerability profile both in terms of discontinuation and the amount of side effects comparing to sertraline. Relatively small number of patients, open-labeled design, lack of randomization and placebo-control are the main limitations of this study that limit the generalizability of the findings and further studies without these limitations are needed. Lack of assessing laboratory tests like blood liver enzymes is another limitation of this study that particularly agomelatine is associated with elevation of hepatic enzymes. ${ }^{26)}$

\section{REFERENCES}

1. Loo H, Hale A, D'haenen H. Determination of the dose of agomelatine, a melatonergic agonist and selective 5-HT(2C) antagonist, in the treatment of major depressive disorder: a placebo-controlled dose range study. Int Clin Psychopharmacol 2002;17:239-247.

2. Goodwin GM. Clinical studies on the efficacy of agomelatine on depressive symptoms. CNS Drugs 2009;23 Suppl 2:35-39.

3. Olié JP, Kasper S. Efficacy of agomelatine, a MT1/MT2 receptor agonist with 5-HT2C antagonistic properties, in major depressive disorder. Int $J$ Neuropsychopharmacol 2007; 10:661-673.

4. Guaiana G, Gupta S, Chiodo D, Davies SJ, Haederle K, Koesters M. Agomelatine versus other antidepressive agents for major depression. Cochrane Database Syst Rev 2013; (12):CD008851.

5. Demyttenaere K, Corruble E, Hale A, Quera-Salva MA, Picarel-Blanchot F, Kasper S. A pooled analysis of six month comparative efficacy and tolerability in four randomized clinical trials: agomelatine versus escitalopram, fluoxetine, and sertraline. CNS Spectr 2013;18:163-170.

6. Shu L, Sulaiman AH, Huang YS, Fones Soon Leng C, Crutel VS, Kim YS. Comparable efficacy and safety of 8 weeks treatment with agomelatine 25-50mg or fluoxetine 20-40mg in Asian out-patients with major depressive disorder. Asian J Psychiatr 2014;8:26-32.

7. Corruble E, de Bodinat C, Belaïdi C, Goodwin GM; Agomelatine Study Group. Efficacy of agomelatine and escitalopram on depression, subjective sleep and emotional experiences in patients with major depressive disorder: a 24-wk randomized, controlled, double-blind trial. Int J Neuropsychopharmacol 2013;16:2219-2234.

8. Montgomery SA, Asberg M. A new depression scale designed to be sensitive to change. $\mathrm{Br} J$ Psychiatry 1979; 134:382-389.

9. Torun F, Önder ME, Torun SD, Tural Ü, Şişmanlar ŞG. The reliability and validity of Montgomery-Asberg depression rating scale for Turkish version. 3P Dergisi 2002;10: 319-330. Turkish.

10. Busner J, Targum SD. The clinical global impressions scale: applying a research tool in clinical practice. Psychiatry (Edgmont) 2007;4:28-37.

11. Lingjaerde O, Ahlfors UG, Bech P, Dencker SJ, Elgen K. The UKU Side Effect Rating Scale: A new comprehensive rating scale for psyhotropic drugs and a cross-sectional study of side effects in neuroleptic-treated patients. Acta Psychiatr Scand 1987;76:1-100.

12. Kennedy SH, Rizvi S, Fulton K, Rasmussen J. A doubleblind comparison of sexual functioning, antidepressant efficacy, and tolerability between agomelatine and venlafaxine XR. J Clin Psychopharmacol 2008;28:329-333.

13. Komaram RB, Nukala S, Palla J, Nambaru LR, Kasturi SM. A comparative study of efficacy and safety of agomelatine and escitalopram in major depressive disorder. J Clin Diagn Res 2015;9:VC05-VC08.

14. Martinotti G, Sepede G, Gambi F, Di Iorio G, De Berardis $\mathrm{D}$, Di Nicola M, et al. Agomelatine versus venlafaxine XR in the treatment of anhedonia in major depressive disorder: a pilot study. J Clin Psychopharmacol 2012;32:487-491.

15. Kasper S, Corruble E, Hale A, Lemoine P, Montgomery SA, Quera-Salva MA. Antidepressant efficacy of agomelatine versus SSRI/SNRI: results from a pooled analysis of headto-head studies without a placebo control. Int Clin Psychopharmacol 2013;28:12-19.

16. Hale A, Corral RM, Mencacci C, Ruiz JS, Severo CA, Gentil V. Superior antidepressant efficacy results of agomelatine versus fluoxetine in severe MDD patients: a randomized, double-blind study. Int Clin Psychopharmacol 2010;25:305-314.

17. Quera-Salva MA, Lemoine P, Guilleminault C. Impact of the novel antidepressant agomelatine on disturbed sleep-wake cycles in depressed patients. Hum Psychopharmacol 2010; 25:222-229.

18. Lemoine P, Guilleminault C, Alvarez E. Improvement in subjective sleep in major depressive disorder with a novel antidepressant, agomelatine: randomized, double-blind comparison with venlafaxine. J Clin Psychiatry 2007;68:17231732.

19. Quera-Salva MA, Hajak G, Philip P, Montplaisir J, Keufer-Le Gall S, Laredo J, et al. Comparison of agomelatine and escitalopram on nighttime sleep and daytime condition and efficacy in major depressive disorder patients. Int Clin Psychopharmacol 2011;26:252-262.

20. Avila A, Cardona X, Martin-Baranera M, Leon L, Caballol N, Millet P, et al. Agomelatine for depression in parkinson disease: additional effect on sleep and motor dysfunction. J Clin Psychopharmacol 2015;35:719-723.

21. Huang KL, Lu WC, Wang YY, Hu GC, Lu CH, Lee WY, et al. Comparison of agomelatine and selective serotonin reuptake inhibitors/serotonin-norepinephrine reuptake inhibitors in major depressive disorder: a meta-analysis of head-to-head randomized clinical trials. Aust $N Z J$ Psychiatry 2014;48:663-671.

22. Llorca PM. The antidepressant agomelatine improves the 
quality of life of depressed patients: implications for remission. J Psychopharmacol 2010;24:21-26.

23. Stahl SM, Fava M, Trivedi MH, Caputo A, Shah A, Post A. Agomelatine in the treatment of major depressive disorder: an 8-week, multicenter, randomized, placebo-controlled trial. J Clin Psychiatry 2010;71:616-626.

24. Goodwin GM, Emsley R, Rembry S, Rouillon F; Agomelatine Study Group. Agomelatine prevents relapse in patients with major depressive disorder without evidence of a discontinuation syndrome: a 24-week randomized, double- blind, placebo-controlled trial. J Clin Psychiatry 2009;70: 1128-1137.

25. Heun R, Ahokas A, Boyer P, Giménez-Montesinos N, Pontes-Soares F, Olivier V, et al. The efficacy of agomelatine in elderly patients with recurrent major depressive disorder: a placebo-controlled study. J Clin Psychiatry 2013;74:587-594.

26. Gahr M, Kratzer W, Fuchs M, Connemann BJ. Safety and tolerability of agomelatine: focus on hepatotoxicity. Curr Drug Metab 2014;15:694-702. 